\title{
Closing and Opening based on discrete t-norms. Applications to natural Image Analysis
}

\author{
M. González-Hidalgo ${ }^{1}$ S. Massanet ${ }^{1}$ \\ ${ }^{1}$ Dept. of Math. and Comp. Science, University of the Balearic Islands, 07122 Palma de Mallorca, Spain
}

\begin{abstract}
This paper delves deeply into the recently introduced mathematical morphology based on discrete t-norms. Closing and opening operators and the concepts of open and closed objects are introduced. All the properties satisfied by nilpotent t-norms, even the generalized idempotence, hold too. After that, some experimental results, using comparative measures, on edge detection are showed. Some experimental results concerning the Top-Hat transformations and the basic filters built from the opening and closing operators are presented. Top-Hat experiments are compared with those obtained with the umbra approach, nilpotent t-norms and uninorms, proving that the discrete approach provides notable results. Moreover, different objective measures are used in order to evaluate the filtered results depending on the amount of noise in the image.
\end{abstract}

Keywords: Discrete t-norm, mathematical morphology, edge detection, noise reduction.

\section{Introduction}

The fuzzy mathematical morphology is a generalization of the binary morphology ([1]) using concepts and techniques from the fuzzy sets theory $([2],[3],[4])$. This theory allows a better treatment and a representation with greater flexibility of the uncertainty and ambiguity present in any level of an image (raw sensor output and its extension to higher levels). Consequently, the component extraction and the shape recognition improve drastically. The four basic morphological operators are dilation, erosion, closing and opening. Thanks to the fact that gray-scale images can be represented as fuzzy sets, fuzzy tools can be used to define fuzzy morphological operators. Thus, conjuntors (usually continuous t-norms) and its residual implicators has been used. Conjunctive uninorms also recently have proved useful as a special case of conjunctors (see [5], [6], [7]).

However, gray-scale images are not represented in practice as functions of $\mathbb{R}^{n}$ into $[0,1]$ because they are stored in finite matrices whose gray levels belong to a finite chain of 256 values. Therefore, the images are represented as discrete functions and discrete fuzzy operators can be used. In [8] it was shown that it is possible to use discrete t-norms to build a fuzzy mathematical morphology that satisfies all the classical properties. Initial results in edge detection, showed there, improved, at least at naked eye, those obtained using the nilpotent t-norms. The mathematical morphology has been already used in medical image analysis ([9], [10]). Therefore, discrete fuzzy mathematical morphology could have a wide range of applications in this research area. The use of performance measures supports this fact since it shows that the discrete framework outperforms the currently used mathematical morphologies and in some cases, the classical algorithms (Canny, Sobel, Prewitt...). However, medical images can be distorted by noise and consequently, morphological filters will play a key role (see [9], [11]). In this direction, the study of the algebraic properties and characterization of the closing and opening discrete operators and open and closed objects when using discrete t-norms is indispensable. Properties obtained are similar to those obtained in [12] and in a broader context, in [13]. This theoretical background allows the construction of the so-called alternate filters and the Top-Hat transformations. The Top-Hat is used to highlight certain components of the image, while the alternate filters are designed to eliminate and reduce noise.

The communication is organized as follows. In Section 2, definitions and properties of fuzzy discrete morphological operators are recalled. In Section 3 the properties related to open and closed objects including the generalized idempotence law are presented. In Section 4, some experimental results on medical images edge detection are performed. Then, the Top-Hat transformation is introduced and results comparing different morphological approaches are shown. The behaviour of alternate filters is investigated depending on the structuring element shape and the amount of noise in the image. Some different objective measures together with the fuzzy $D I$-subsethood measures ([14]) are used to evaluate the filtered results.

\section{Fuzzy discrete morphological operators}

We will suppose the reader to be familiar with the basic definitions and properties of the fuzzy discrete logical operators that will be used in this work, specially those related to discrete t-norms and discrete residual implicators (see [8]). From now on, the following notation will be used: $L=\{0, \ldots, n\}$ a finite chain, $\mathcal{I}$ will denote a discrete implicator, $\mathcal{C}$ a dis- 
crete conjunctor, $\mathcal{N}$ the only strong negation on $L$ which is given by $N(x)=n-x$ for all $x \in L, T$ a discrete t-norm, $\mathcal{I}_{T}$ its residual implicator, $A$ a gray-scale image and $B$ a gray-level structuring element that takes values on L. Our methodology is similar to the used ones in $[3,4,5,12,13]$.

Definition 1 The fuzzy discrete dilation $D_{\mathcal{C}}(A, B)$ and the fuzzy discrete erosion $E_{\mathcal{I}}(A, B)$ of $A$ by $B$ are the gray-scale images defined as

$$
\begin{aligned}
& D_{\mathcal{C}}(A, B)(y)=\max _{x} \mathcal{C}(B(x-y), A(x)) \\
& E_{\mathcal{I}}(A, B)(y)=\min _{x} \mathcal{I}(B(x-y), A(x)) .
\end{aligned}
$$

Definition 2 The fuzzy discrete closing $C_{\mathcal{C}, \mathcal{I}}(A, B)$ and the fuzzy discrete opening $O_{\mathcal{C}, \mathcal{I}}(A, B)$ of $A$ by $B$ are the gray-scale images defined as

$$
\begin{aligned}
C_{\mathcal{C}, \mathcal{I}}(A, B)(y) & =E_{\mathcal{I}}\left(D_{\mathcal{C}}(A, B),-B\right)(y) \\
O_{\mathcal{C}, \mathcal{I}}(A, B)(y) & =D_{\mathcal{C}}\left(E_{\mathcal{I}}(A, B),-B\right)(y) .
\end{aligned}
$$

The reflection $-B$ of an $n$-dimensional fuzzy set $B$ is defined as $-B(x)=B(-x)$, for all $x \in \mathbb{Z}^{n}$.

Obviously a discrete t-norm is a conjunctor. Thus, these operators and their residual implicators can be used to define fuzzy discrete morphological operators using the previous definitions guaranteeing the adjunction property. In [8], the discrete tnorms that have to be used in order to preserve the morphological and algebraic properties that satisfy the classical morphological operators were fully determined. The algebraic properties of the fuzzy discrete morphological operators that will be used in the next section are recalled.

- The fuzzy dilation $D_{T}$ is increasing in both arguments, the fuzzy erosion $E_{\mathcal{I}_{T}}$ is increasing in the first argument and decreasing in the second one, the fuzzy closing $C_{T, \mathcal{I}_{T}}$ and the fuzzy opening $O_{T, \mathcal{I}_{T}}$ are both increasing in the first argument.

- All the usual properties (those satisfied in the morphology based on left-continuous t-norms) respect to the interactions with Zadeh's union and intersection of discrete images are also satisfied.

- If $B(0)=n$ we have: $E_{\mathcal{I}_{T}}(A, B) \subseteq A \subseteq$ $D_{T}(A, B)$.

- In addition, $O_{T, \mathcal{I}_{T}}(A, B) \subseteq A \subseteq C_{T, \mathcal{I}_{T}}(A, B)$.

- The fuzzy closing and the fuzzy opening are idempotent, i.e.: $\quad C_{T, \mathcal{I}_{T}}\left(C_{T, \mathcal{I}_{T}}(A, B), B\right)=$ $C_{T, \mathcal{I}_{T}}(A, B)$ and $O_{T, \mathcal{I}_{T}}\left(O_{T, \mathcal{I}_{T}}(A, B), B\right)=$ $O_{T, \mathcal{I}_{T}}(A, B)$.

- If $B(0)=n$, then $E_{\mathcal{I}_{T}}(A, B) \subseteq O_{T, \mathcal{I}_{T}}(A, B) \subseteq$ $A \subseteq C_{T, \mathcal{I}_{T}}(A, B) \subseteq D_{T}(A, B)$.

- As in classical morphology, the difference between the fuzzy dilation and the fuzzy erosion of a gray-scale image, $D_{T}(A, B) \backslash E_{\mathcal{I}_{T}}(A, B)$, called the fuzzy gradient operator, can be used in edge detection.
In addition to these properties, it is worth to safeguard the duality between the discrete fuzzy morphological operators. Therefore, discrete t-norms satisfying $\mathcal{I}_{T}=\mathcal{I}_{T, \mathcal{N}}$ are needed. This property holds for the discrete t-norms enumerated in the following result (see [8]).

Proposition 3 The identity $\mathcal{I}_{T}=\mathcal{I}_{T, \mathcal{N}}$ is satisfied in the following cases:

1. When $T$ is the Eukasiewicz discrete t-norm, $T_{L}(x, y)=\max \{0, x+y-n\}$.

2. When $T$ is the nilpotent minimum given by the following expression

$$
T_{n M}(x, y)= \begin{cases}0 & \text { if } x+y \leq n \\ \min \{x, y\} & \text { otherwise }\end{cases}
$$

3. When $T$ is an ordinal sum (with only one summand) of the Eukasiewicz t-norm in a square $[a, n-a]^{2}, a \in L$ with $a \leq n-a$, truncated by 0 , given by the expression

$$
\begin{aligned}
& T_{n M a}(x, y)= \\
& \begin{cases}0 & \text { if } x+y \leq n \\
x+y-(n-a) & \text { if } x+y>n \text { and } \\
\min \{x, y\} & a<x, y \leq n-a\end{cases}
\end{aligned}
$$

\section{Closed and open fuzzy objects}

The idempotence properties of fuzzy opening and closing when $T$ is a discrete t-norm and $\mathcal{I}_{T}$ its residual implicator motivate, as in the classical mathematical morphology, the following definitions.

Definition 4 Let $A$ and $B$ be two gray-scale images. It is said that $A$ is $B$-closed (resp. B-open) if $C_{T, \mathcal{I}_{T}}(A, B)=A\left(\right.$ resp. $\left.O_{T, \mathcal{I}_{T}}(A, B)=A\right)$.

It is important to note that due to the idempotence of the closing and opening, the closing is $B$ closed and the opening is $B$-open. Almost all the results presented in this section are analogous to the respective in the $[0,1]$-framework and the proofs are similar and therefore, they are not included (see [12] and [7]). The only difference worth to mention is related to the left-continuity or the right-continuity of the conjunctor or the implicator. These properties are necessary in the $[0,1]$-framework in order to ensure that both operators preserve the infimum and the supremum adequately. However, in the discrete approach they are not necessary since we work with maxima and minima. First of all, each $B$-open and $B$-closed objects are the opening or closing of some image, respectively.

Proposition 5 Let $T$ be a discrete t-norm and $\mathcal{I}_{T}$ its residual implicator. Then it holds that $A$ is $B$ open (resp. B-closed) if, and only if, it exists a fuzzy object $F$ such that $A=D_{T}(F,-B)$ (resp. $A=$ $\left.E_{\mathcal{I}_{T}}(F,-B)\right)$. 
In [13], it is stated that the closing and opening operators only make sense if the opening always gives an open object, and the closing gives a closed object. Furthermore, it is recommended that they have extrema properties. The following result shows that these requisites hold in this theory.

Proposition 6 Let $T$ be a discrete t-norm and $\mathcal{I}_{T}$ its residual implicator. Then $O_{T, \mathcal{I}_{T}}(A, B)$ $i s$ the largest $B$-open subset greater than $A$ and $C_{T, \mathcal{I}_{T}}(A, B)$ is the smallest $B$-closed subset that contains $A$.

The intersections and unions preserve the properties of $B$-open and $B$-closed objects, respectively.

Proposition 7 Let $T$ be a discrete t-norm and $A_{1}$ and $A_{2}$ two gray-scale images. Then if $A_{1}$ and $A_{2}$ are both $B$-open (resp. B-closed) then $A_{1} \cup A_{2}$ is $B$-open (resp. $A_{1} \cap A_{2}$ is $B$-closed).

Up to now any discrete t-norm satisfies all the properties. However, if the duality between fuzzy open and closed objects is required, we must resort to the discrete t-norms of Proposition 3.

Proposition 8 Let $T$ be a discrete t-norm of Proposition 3. Then, $A$ is B-open if, and only if, co $A$ is $B$-closed, where $($ co $A)(x)=n-A(x)$.

One of the most important properties that the morphological operators can satisfy is the so-called generalized idempotence. This property is satisfied by the classical opening and closing and the fuzzy ones when we consider continuous t-norms and their residual implicator. Using the discrete tnorms of Proposition 3 and some similar inequalities to the ones in Propositions 10-13 in [7] for discrete t-norms, the generalized idempotence of the discrete fuzzy opening and closing is proved in a similar way to the $[0,1]$ case. It is obvious that in the discrete case, there is no restriction on the ranges of $A$ and $B$.

Proposition 9 Let $T$ be a discrete t-norm of those of Proposition 3 and $\mathcal{I}_{T}$ its residual implicator. If $A$ is B-open, then for all fuzzy object $F$, $O_{T, \mathcal{I}_{T}}(F, A) \subseteq O_{T, \mathcal{I}_{T}}(F, B) \subseteq F$ holds and by duality, $F \subseteq C_{T, \mathcal{I}_{T}}(F, B) \subseteq C_{T, \mathcal{I}_{T}}(F, A)$.

Proposition 10 (Generalized idempotence) Let $T$ be a discrete $t$-norm of those of Proposition 3 and $\mathcal{I}_{T}$ its residual implicator. If $A$ is $B$-open, then for all fuzzy object $F, O_{T, \mathcal{I}_{T}}\left(O_{T, \mathcal{I}_{T}}(F, B), A\right)=$ $O_{T, \mathcal{I}_{T}}\left(O_{T, \mathcal{I}_{T}}(F, A), B\right)=O_{T, \mathcal{I}_{T}}(F, A)$ holds and dually for the closing.

Remark 11 The two previous propositions are valid for any discrete t-norm. However, due to the fact that duality is only satisfied when using the discrete t-norms of Proposition 3, these are the only ones that satisfy all the algebraic properties to guarantee a good mathematical morphology.
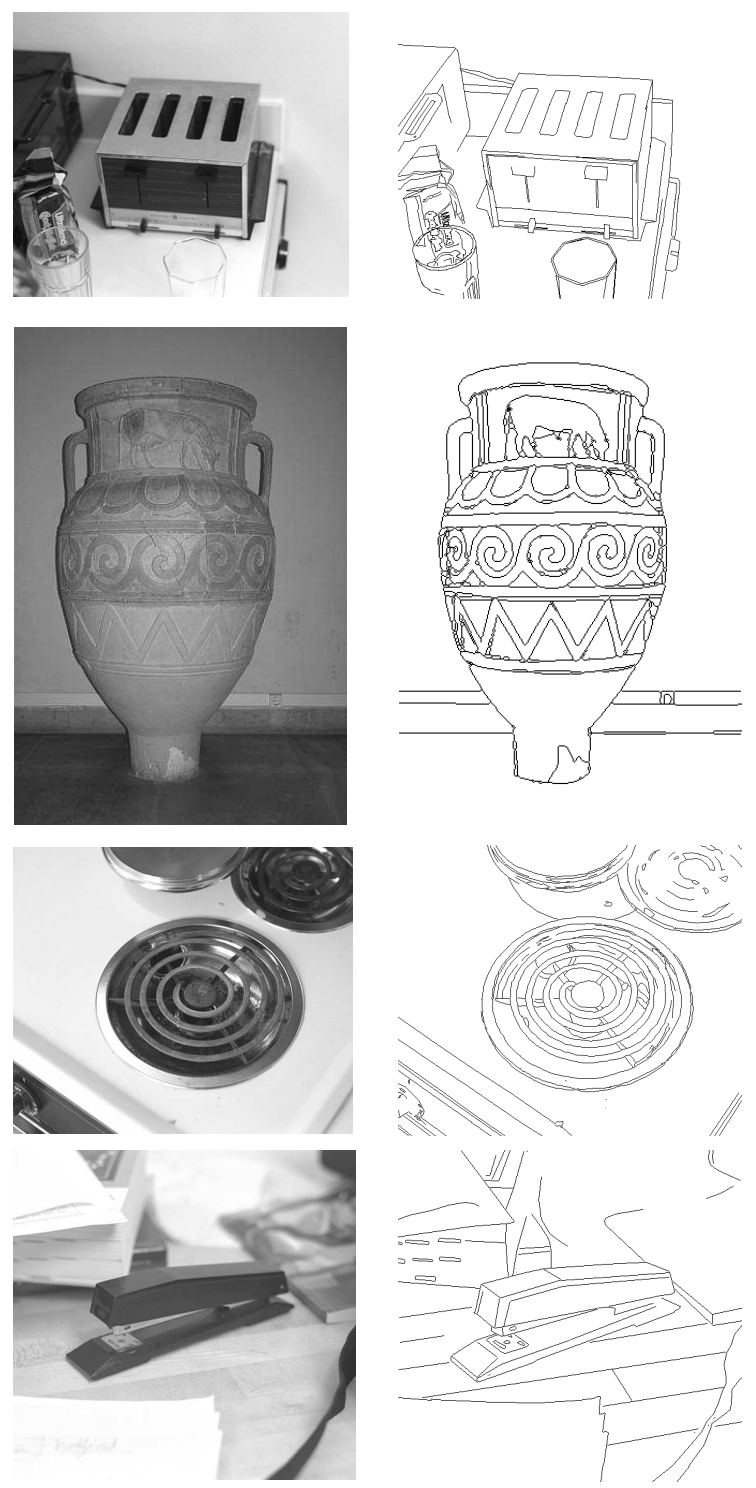

Figure 1: Original images used in the edge detection test (left) and their ground-truth (right)

\section{Experimental results}

\subsection{Edge detection}

Edge detection is a fundamental pre-processing step in applications such as image segmentation and computer vision, and its performance is relevant for the final results of the image processing. In particular, medical images edge detection is an indispensable step in medical image segmentation and 3D reconstruction. As we have already said, the fuzzy morphological gradient

$$
D_{T}(A, B) \backslash E_{\mathcal{I}_{T}}(A, B)
$$

is a useful tool for edge detection. In [8], some initial results on edge detection were presented. Those results proved that the discrete approach give very competitive results if we compare them with those obtained from the umbra approach, nilpotent tnorms or uninorms. However, some quantitative 

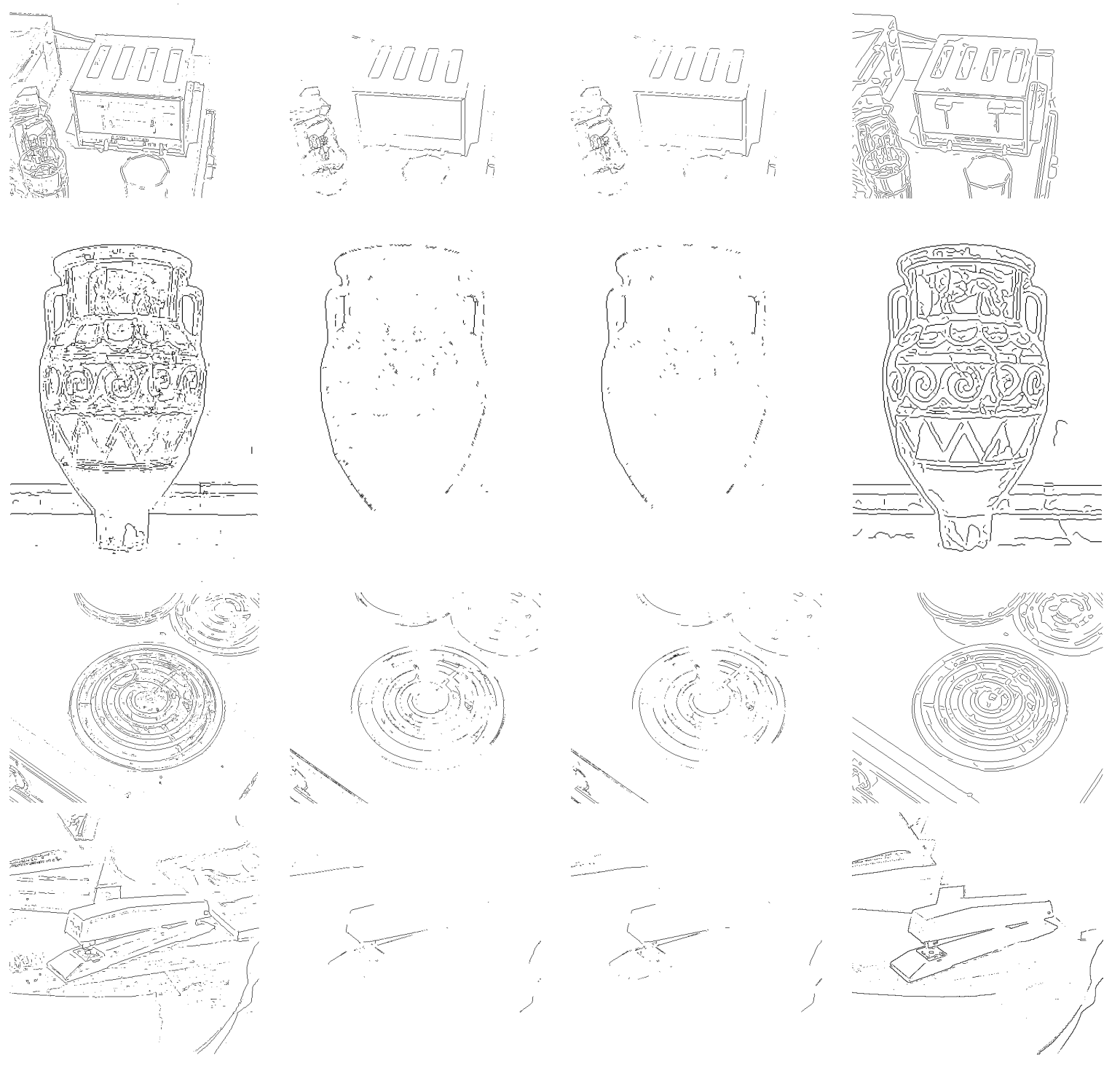

Figure 2: From left to right, binarized edge maps obtained by nilpotent minimum, nilpotent Łukasiewicz t-norm, umbra approach and best result of classical algorithms.

objective performance measure is needed in order to ensure this statement instead of the simple observation of the results at naked eye. In this way, it is necessary to have some natural images with their ground truth (edges specification) as a dataset from which to compare the outputs by the different algorithms with the use of a performance measure. Thus, the images and their ground-truth from Figure 1, belonging to the Berkeley Segmentation Dataset ([15]) and the public dataset of the University of South Florida ([16]), have been used. In order to quantify the similarity between the detected edges (DE) and the ground truth (GT), we use the widely used Pratt's figure of merit (FoM, [17]), defined as

$$
F_{o M}=\frac{1}{\max \{\operatorname{card}\{D E\}, \operatorname{card}\{G T\}\}} \cdot \sum_{x \in D E} \frac{1}{1+a d^{2}},
$$

where card is the number of edge points of the image, $a$ is a scaling constant and $d$ is the separation distance of an actual edge point to the ideal edge points. FoM takes values in $(0,1]$, being equal to 1 if, and only if, DE coincides with GT, and is nonsymmetric with respect to DE and GT. This measure provides an overall evaluation of the quality of a given contour map, by taking into account both the amount of false positive (undesired presences on the edge map), false negative (missing edges) and shifting or deformation of a correctly detected edges from its GT position.

These images are applied to the morphological operators based on discrete t-norms established in the previous section. In all the experimental results, we applied the nilpotent minimum with the following structuring element, already used in [4],

$$
B=\left(\begin{array}{lll}
219 & 219 & 219 \\
219 & 255 & 219 \\
219 & 219 & 219
\end{array}\right) .
$$

The obtained results are compared through the 

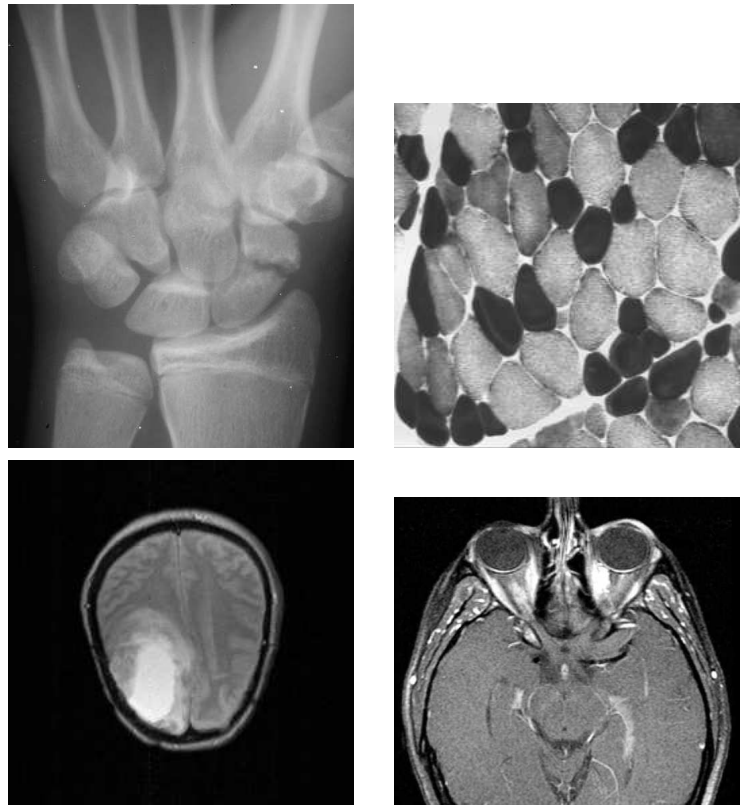

Figure 3: Original images used in the edge detection experimental results

computation of the FoM with those obtained by the nilpotent Łukasiewicz t-norm, the umbra approach and the classical edge detection algorithms of Canny, Sobel, Prewitt, Roberts, Laplacian of Gaussian operator (LoG) implemented in Matlab R2008a. For Canny, we modify $\sigma$ in order to obtain the best result. We use the counterpart of $B$ in $[0,1]$ as structuring element for the Łukasiewicz t-norm. In order to use FoM, the images must be binarized. In this way, the best result of non-maxima suppression ([18]) with experimentally obtained threshold, and the Otsu thresholding method ([19]) with the thinning algorithm implemented in Matlab are applied in the morphological approaches (the default ones for the classical algorithms). In Figure 2, we show the results obtained by the discrete approach, the nilpotent Łukasiewicz t-norm, the umbra approach and the best of the classical algorithms of the images displayed in Figure 1. In Table 1, the values of FoM for each image and each algorithm are presented.

Note that in all four images, FoM gives the discrete approach a better mark. Although the values are quite similar, it is important to remark that the discrete framework could have a wide margin of improvement because in these experiments, we have only used a fixed structuring element with a fixed discrete t-norm (the nilpotent minimum). Better results could be obtained using an adequate structuring element for each image, or applying one t-norm of the family $T_{3}$ of Proposition 3 that are between the nilpotent minimum and the Eukasiewicz t-norm. On the other hand, Canny's method detects too much (in particular, textures) for small values of sigma, meanwhile the geometry of the shape of the object is changed for greater values of sigma. Thus,
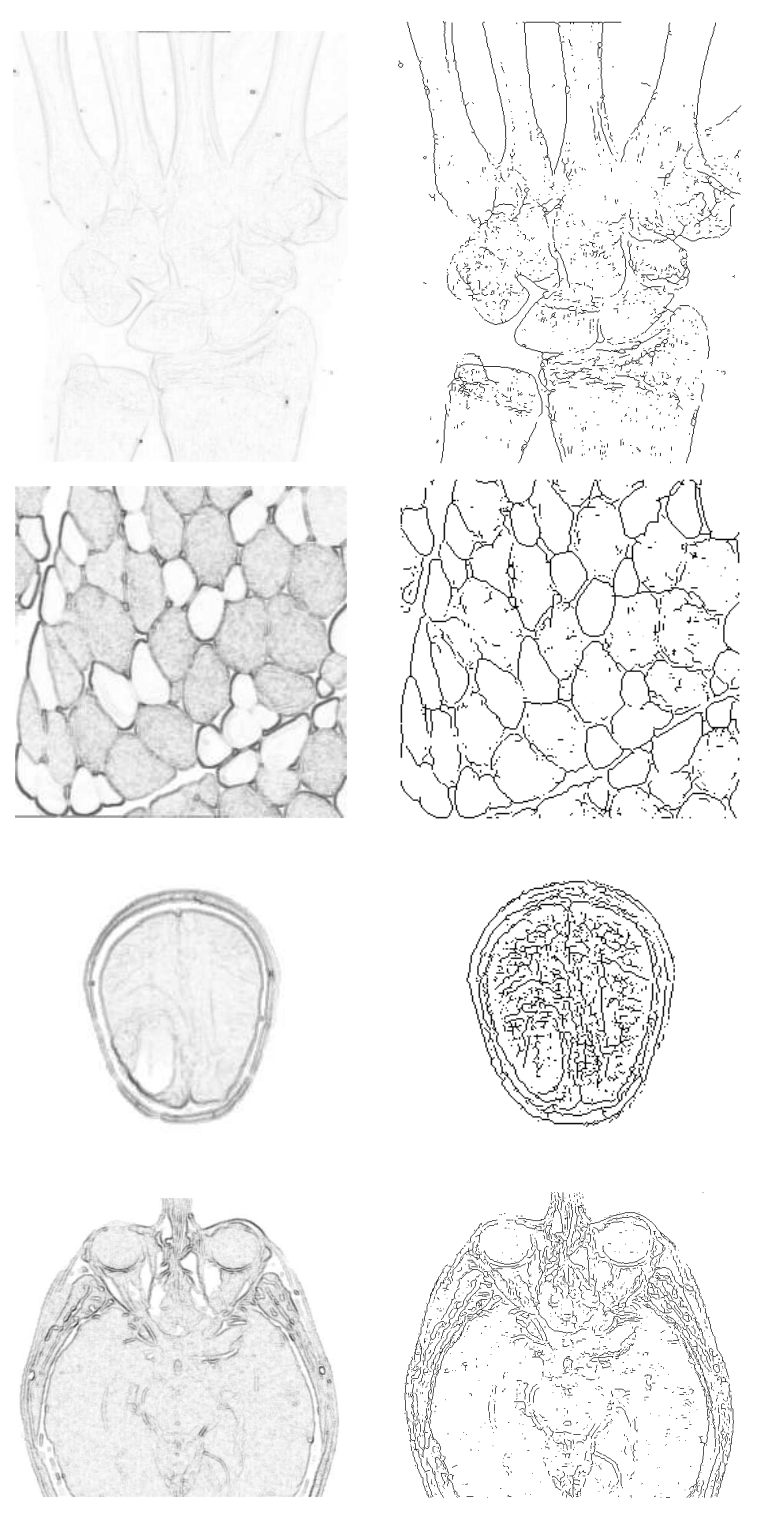

Figure 4: Detected gray-scale edges (left). Binarized with a threshold (right)

FoM decreases its value in both cases. From the edge contours detected, it is noticeably the improvement achieved with the use of discrete t-norms in respect to the results obtained with the only t-norm on $[0,1]$ satisfying all the desirable algebraic properties, the Eukasiewicz t-norm. Note that this approach gives similar results to those of the umbra approach.

In order to check the applications of the discrete fuzzy mathematical morphology to medical image analysis, we applied the edge detector to the images of Figure 3. There we have a fracture on the escaphoid, some muscle cells, a brain tumor and a head tomography. The results obtained are displayed in Figure 4. For each image, the gray-scale edge image is obtained and then an experimentally obtained threshold is applied in order to get the binary edges. Also these edges are refined with the non-maxima suppression algorithm. 
Table 1: Values of FoM for the results obtained for each algorithm for each image displayed in Figure 1

\begin{tabular}{|l|r|r|r|r|r|r|r|r|}
\hline Image & Nilp. Min. & Lukasiewicz & Umbra App. & Canny & Sobel & Prewitt & Roberts & LoG \\
\hline \hline Roaster & 0.4978 & 0.2603 & 0.2239 & 0.4934 & 0.4820 & 0.4838 & 0.4425 & 0.4633 \\
\hline Vase & 0.4529 & 0.0786 & 0.0554 & 0.3989 & 0.3898 & 0.3877 & 0.3435 & 0.3784 \\
\hline Burner & 0.4843 & 0.2734 & 0.2117 & 0.4732 & 0.4688 & 0.4610 & 0.4393 & 0.4273 \\
\hline Stapler & 0.3978 & 0.0644 & 0.0836 & 0.3693 & 0.3683 & 0.3672 & 0.3696 & 0.2831 \\
\hline
\end{tabular}

\subsection{Basic and alternate filters. Top-Hat transformations}

The discrete opening and closing previously defined are the elementary morphological filters, or basic filters. Due to the fact that the opening is a non-extensive filter and the opening an extensive one, they can be used to suppress non-desired objects in an image. The opening of a gray-scale image by means of an structuring element deletes the light zones smaller than the structuring element and darkens the light objects. On the other hand, the closing removes the dark zones smaller than the structuring element, enlightening the dark objects. Thus, the size and shape of the structuring element are key factors to remove non-desired objects of an image taking into account that the remaining structures should be not affected.

The opening and closing are morphological transformations and because of that they are useful to compute their associated residuals, known as TopHat transformations. These transformations find structures which have been removed by the opening and closing filters and the residual between the original image and the filtered image increases notably the contrast of the erased regions (see [1]). So, the Top-Hat transformations are defined as follows

$$
\begin{aligned}
& \rho_{T, \mathcal{I}_{T}}(A, B)=A \backslash O_{T, \mathcal{I}_{T}}(A, B) \text { (Top-Hat) } \\
& \rho_{T, \mathcal{I}_{T}}^{d}(A, B)=C_{T, \mathcal{I}_{T}}(A, B) \backslash A \text { (Dual Top-Hat). }
\end{aligned}
$$

The Top-Hat transformation enhances the light objects that have been removed by the opening and it is used to extract contrasted components from the background, while the dual Top-Hat extracts the dark components which have been removed by the closing. Usually, these transformations delete the soft trends.

In Figure 5, a comparison of both transformations for the different morphological approaches is displayed. We compare the results obtained by the discrete approach with those obtained by the umbra approach and by the Eukasiewicz continuous tnorm. In addition, they are compared with those obtained using as conjunctor an idempotent uninorm derived of the negation $\mathcal{N}(x)=1-x$ (see $[7])$. Recall that for the morphology based on continuous t-norms we use the counterpart of (1) on $[0,1]$ and scaled by $e=0,5$ in the uninorm framework, where $e$ is the neutral element of the uninorm. In the results, a threshold has been applied in order to better visualize them. As we can observe,

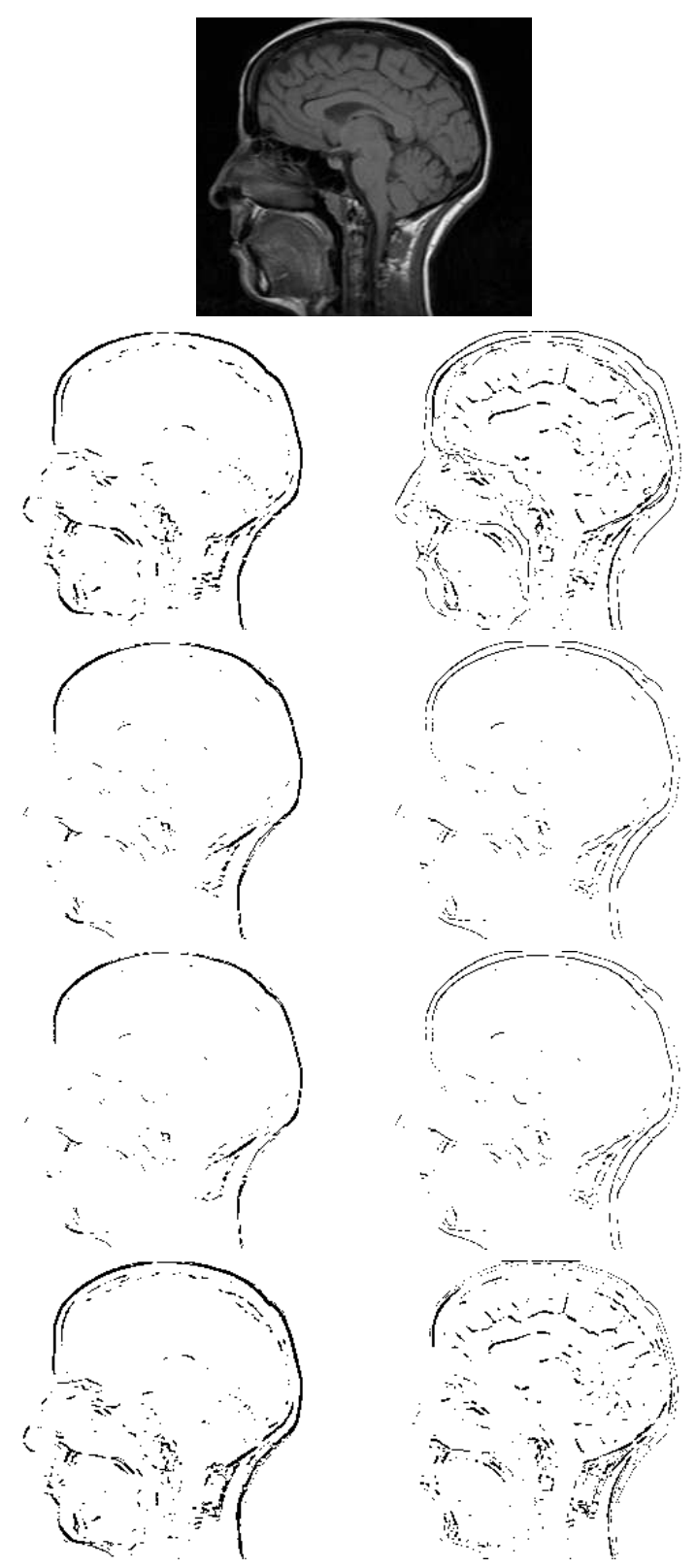

Figure 5: Top-Hat (left) and Dual Top-Hat (right) using from top to bottom, $T_{n M}$, continuous $T_{L}$, umbra approach and idempotent uninorm of the original image on the first row

the discrete t-norms outperform at naked eye the results obtained by the other morphologies.

As we have already commented, the closing and the opening are the basic filters of the fuzzy mathematical morphology. The composition or combination of these operators between them is the most 

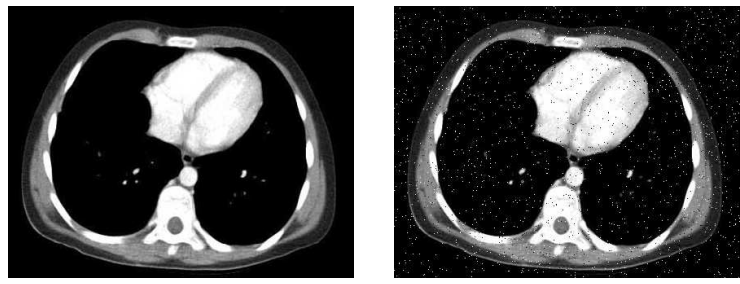

Figure 6: Original medical image (left) with salt and pepper noise added (right) used in the noise reduction experiments.

used way to build new filters $([1])$. The initial filters that one can build from the opening and closing are the alternate filters. Let $\xi(A, B)$ and $\psi(A, B)$ be the opening and closing, respectively, of an image $A$ by an structuring element $B$ using a discrete t-norm as conjunctor and its residual implicator. Now, we can build four idempotent and increasing filters: $\xi \psi, \psi \xi$, $\psi \xi \psi$ and $\xi \psi \xi$. Due to the idempotence property, the composition of more than three operators does not provide us new filters.

In Figure 6, we have a chest tomography original image and the same image with added salt and pepper noise with parameter 0.02. The noise was added using the standard functions of Matlab R2008a. As in the case of edge detection, different objective measures can be used to evaluate the performance of a filter. Among them, we will use the MSE, the SNR, the SSIM (see [20]) and the fuzzy $D I$-subsethood measure $E Q_{\sigma D I}$ (see [14]). Let $O_{1}$ and $F_{2}$ be two images of dimensions $M \times N$. We suppose that $O_{1}$ is the original noise-free image and $F_{2}$ is the restored image for which some filter has been applied. The $E Q_{\sigma D I}$ values are defined as follows:

$E Q_{\sigma D I}\left(F_{2}, O_{1}\right)=\frac{1}{M N} \sum_{i=1}^{M} \sum_{j=1}^{N}\left(1-\left|O_{1}(i, j)-F_{2}(i, j)\right|\right)$.

Smaller values of MSE and larger values of SNR, SSIM and $E Q_{\sigma D I}\left(0 \leq S S I M, E Q_{\sigma D I} \leq 1\right)$ are indicators of better capabilities for noise reduction and image recovery.

Figure 7 shows the effect of the choice of different discrete t-norms in the results as well as how the measures are improved using a more adequate structuring element for this type of noise as $B_{2}$ (see Table 2), where

$$
B_{2}=\left(\begin{array}{ccc}
0 & 255 & 0 \\
255 & 255 & 255 \\
0 & 255 & 0
\end{array}\right)
$$

Note that all the measures obtained with $B_{2}$ are better than the ones obtained with $B$. In addition, there is no consensus between the measures to the question of which t-norm performs better when using $B\left(E Q_{\sigma D I}\right.$ gives to Łukasiewicz t-norm the better mark while the others give it to $\left.T_{n M 25}\right)$. For binary structuring elements as $B_{2}$ all discrete t-norms give the same result.
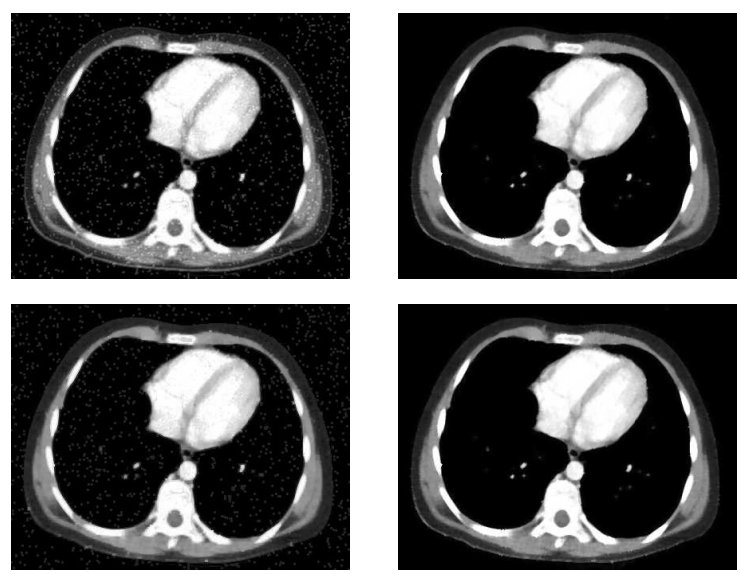

Figure 7: $\psi \xi \psi$ filter using in descending order discrete $T_{L}$ and $T_{n M}$ with $B$ (left) and $B_{2}$ (right)

Table 2: Values of performance for the results obtained for each discrete t-norm in Figure 7

\begin{tabular}{|l|c|r|r|r|r|}
\hline t-norm & S.E. & MSE & SNR & SSIM & $E Q_{\sigma D I}$ \\
\hline \hline \multirow{2}{*}{$T_{L}$} & $B$ & 156.291 & 16.6852 & 0.988369 & 0.9843 \\
\cline { 2 - 6 } & $B_{2}$ & 28.8755 & 24.0193 & 0.997982 & 0.9941 \\
\hline \multirow{2}{*}{$T_{n M}$} & $B$ & 134.88 & 17.3251 & 0.990033 & 0.9829 \\
\cline { 2 - 6 } & $B_{2}$ & 28.8755 & 24.0193 & 0.997982 & 0.9941 \\
\hline \multirow{2}{*}{$T_{n M 25}$} & $B$ & 133.529 & 17.3688 & 0.990093 & 0.9840 \\
\cline { 2 - 6 } & $B_{2}$ & 28.8755 & 24.0193 & 0.997982 & 0.9941 \\
\hline
\end{tabular}

Table 3 shows the values of these measures in function of the amount of noise added to the original image in Figure 6. Notice that, when the noise increases, the values of the measures remain acceptable and SSIM and $E Q_{\sigma D I}$ values are still higher than 0.99 .

\section{Conclusions and future work}

In this work, it has been proved that the fuzzy mathematical morphology based on discrete tnorms satisfies all the algebraic properties to be a "good" mathematical morphology, including all the properties that are satisfied for the morphology based on nilpotent t-norms without restrictions on the continuity of the conjunctors. Moreover, an edge detection algorithm based on the fuzzy morphology based on discrete t-norms, derived as a residual operator, has been presented. To evaluate the performance of the edge detectors, comparison experiments with other known approaches were carried out using FoM. The results indicate that this is a good starting point to develop more complex algorithms, such as edge detectors based on directional structuring elements or multi-scale edge detectors. In addition, initial experimental results on the Top-Hat transformations show notable results compared with existing fuzzy mathematical morphologies. Also, we have shown how the alternate filters using discrete t-norms can remove the noise 
Table 3: Values of measures for filtered images with $T_{n M}$ and $B_{2}$ changing the amount of noise added.

\begin{tabular}{|l|r|r|r|r|}
\hline Noise & MSE & SNR & SSIM & $E Q_{\sigma D I}$ \\
\hline \hline 0.02 & 24.0193 & 24.372 & 0.997982 & 0.9941 \\
\hline 0.04 & 41.1312 & 22.4828 & 0.997130 & 0.9933 \\
\hline 0.06 & 51.9862 & 21.4657 & 0.996373 & 0.9926 \\
\hline 0.08 & 68.8897 & 20.2430 & 0.995200 & 0.9919 \\
\hline 0.1 & 98.0487 & 18.7101 & 0.993166 & 0.9907 \\
\hline
\end{tabular}

of an image preserving their structure. Moreover, when we use an structuring element better adapted to the noised image we improve the selected objective measures. The future work will consist on the construction of new filters and on the selection of the size, shape, direction of the structuring element adapted to the noised image and how we can improve performance. Other type of noise should be considered.

\section{Acknowledgement}

Supported by the Government Spanish Grant MTM2009-10320, with FEDER support. The authors would like to thank H. Bustince and his group of the Public University of Navarra for his kindly support.

\section{References}

[1] J. Serra. Image analysis and mathematical morphology, vols. 1, 2. Academic Press, London, 1982,1988.

[2] I. Bloch and H. Maître. Fuzzy mathematical morphologies: a comparative study. Pattern Recognition, 28:1341-1387, 1995.

[3] B. De Baets. Fuzzy morphology: A logical approach. In Uncertainty Analysis in Engineering and Science: Fuzzy Logic, Statistics, and Neural Network Approach, pages 53-68. Kluwer Academic Publishers, Norwell, 1997.

[4] M. Nachtegael and E.E. Kerre. Classical and fuzzy approaches towards mathematical morphology. In Fuzzy techniques in image processing, number 52, chapter 1, pages 3-57. PhysicaVerlag, New York, 2000.

[5] B. De Baets, N. Kwasnikowska, and E. Kerre. Fuzzy morphology based on uninorms. In Proc. of the seventh IFSA World Congress, pages 215-220, Prague, 1997.

[6] M. González, D. Ruiz-Aguilera, and J. Torrens. Algebraic properties of fuzzy morphological operators based on uninorms. In Artificial Intelligence Research and Development, volume 100, pages 27-38, Amsterdam, 2003. IOS Press.

[7] M. González-Hidalgo, A. Mir-Torres, D. RuizAguilera, and J. Torrens. Fuzzy morphology based on uninorms: Image edge-detection.
Opening and closing. In Computational Vision and Medical Image Processing, pages 127-133, London, 2008. Taylor \& Francis Group.

[8] M. González-Hidalgo, S. Massanet, and J.Torrens. Discrete t-norms in a fuzzy mathematical morphology: Algebraic properties and experimental results. In Proc. of FUZZ-IEEE, pages 1194-1201, Barcelona, Spain, 2010.

[9] M. González-Hidalgo, A. Mir Torres, and J. Torrens Sastre. Noisy image edge detection using an uninorm fuzzy morphological gradient. In ISDA'09, pages 1335-1340, Los Alamitos, CA, USA, 2009. IEEE Computer Society.

[10] Z. Yu-qian, G. Wei-hua, Ch. Zhen-cheng, T. Jing-tian, and Li Ling-yun. Medical images edge detection based on mathematical morphology. In Proc. of the 2005 IEEE Engineering in Medicine and Biology, pages 6492-6495, Shanghai, China, 2005.

[11] Y. Xu, J. Zhao, and Y. Jiao. Noisy image edge detection based on multi-scale and multistructuring element order morphology transformation. In CISP '08, Vol. 3, pages 379-383. IEEE Computer Society, 2008.

[12] B. De Baets. Idempotent closing and opening operations in fuzzy mathematical morphology. In Proc. of ISUMA-NAFIPS'95, pages 228-233, Maryland, USA, 1995.

[13] U. Bodenhofer. A unified framework of opening and closure operators with respect to arbitrary fuzzy relations. Soft Computing, 7:220 227, 2003.

[14] H. Bustince, M. Pagola, and E. Barrenechea. Construction of fuzzy indices from fuzzy DIsubsethood measures: application to the global comparison of images. Information Sciences, 177:906-929, 2007.

[15] D. Martin, C. Fowlkes, D. Tal, and J. Malik. A database of human segmented natural images and its application to evaluating segmentation algorithms and measuring ecological statistics. In Proc. 8th ICCV, volume 2, pages 416-423, 2001.

[16] K. Bowyer, C. Kranenburg, and S. Dougherty. Edge detector evaluation using empirical roc curves. Computer Vision and Pattern Recognition, 1:354-359, 1999.

[17] W. K. Pratt. Digital Image Processing. Nueva York, EUA : Wiley, 1991.

[18] J. Canny. A computational approach to edge detection. IEEE Trans. Pattern Anal. Mach. Intell., 8(6):679-698, November 1986.

[19] N. Otsu. A threshold selection method from gray-level histograms. IEEE Trans. on Systems, Man and Cybernetics, 9:62-66, 1979.

[20] Z. Wang, A. C. Bovik, H. R. Sheikh, and E. P. Simoncelli. Image quality assessment: From error visibility to structural similarity. IEEE Transactions on Image Processing, 13(4):600612, 2004. 